OPEN ACCESS

Edited by:

Andrea Tinelli,

Moscow Institute of Physics and

Technology, Russia

Reviewed by:

Jean Dubuisson,

Geneva University Hospitals

(HUG), Switzerland

Alfredo Ercoli,

University of Messina, Italy

*Correspondence:

Hua Lal

LlaiHhua@163.com

Da-Jun Wu

drwudj@126.com

Specialty section:

This article was submitted to Obstetrics and Gynecology, a section of the journal

Frontiers in Medicine

Received: 31 March 2021 Accepted: 02 June 2021 Published: 09 July 2021

Citation:

Yang X-L, Huang N, Wang M-M, Lai H and Wu D-J (2021) Comparison of

Different Lymph Node Staging Schemes for Predicting Survival

Outcomes in Node-Positive Endometrioid Endometrial Cancer Patients. Front. Med. 8:688535. doi: 10.3389/fmed.2021.688535

\section{Comparison of Different Lymph Node Staging Schemes for Predicting Survival Outcomes in Node-Positive Endometrioid Endometrial Cancer Patients}

\author{
Xi-Lin Yang ${ }^{1}$, Nan Huang ${ }^{2}$, Ming-Ming Wang ${ }^{2}$, Hua Lai ${ }^{3 *}$ and Da-Jun Wu ${ }^{1 *}$ \\ 'Department of Radiation Oncology, Chengdu Women's and Children's Central Hospital, School of Medicine, University of \\ Electronic Science and Technology of China, Chengdu, China, ${ }^{2}$ Department of Oncology, The First Affiliated Hospital of \\ Chongqing Medical University, Chongqing, China, ${ }^{3}$ Department of Radiology, Chengdu Women's and Children's Central \\ Hospital, School of Medicine, University of Electronic Science and Technology of China, Chengdu, China
}

Objective: To compare the prognostic predictive performance of six lymph node (LN) staging schemes: American Joint Committee on Cancer (AJCC) N stage, number of retrieved lymph nodes (NRLN), number of positive lymph nodes (NPLN), number of negative lymph nodes (NNLN), lymph node ratio (LNR), and log odds of positive lymph nodes (LODDS) among node-positive endometrioid endometrial cancer (EEC) patients.

Methods: A total of 3,533 patients diagnosed with node-positive EEC between 2010 and 2016 from the Surveillance, Epidemiology, and End Results (SEER) database were retrospectively analyzed. We applied X-tile software to identify the optimal cutoff value for different staging schemes. Univariate and multivariate Cox regression models were used to assess the relationships between different LN schemes and survival outcomes [disease-specific survival (DSS) and overall survival (OS)]. Moreover, Akaike information criterion (AIC) and Harrell concordance index (C-index) were used to evaluate the predictive performance of each scheme in both continuous and categorical patterns.

Results: $\mathrm{N}$ stage (N1/N2) was not an independent prognostic factor for node-positive EEC patients based on multivariate analysis (DSS: $p=0.235$; OS: $p=0.145$ ). Multivariate model incorporating LNR demonstrated the most superior goodness of fit regardless of continuous or categorical pattern. Regarding discrimination power of the models, LNR outperformed other models in categorical pattern (OS: C-index $=0.735$; DSS: C-index $=0.737$ ); however, LODDS obtained the highest C-index in continuous pattern (OS: 0.736; DSS: 0.739).

Conclusions: $\quad N$ stage (N1/N2) was unable to differentiate the prognosis for node-positive EEC patients in our study. However, LNR and LODDS schemes seemed to have a better predictive performance for these patients than other number-based LN schemes whether in DSS or OS, which revealed that LNR and LODDS should be more helpful in prognosis assessment for node-positive EEC patients than AJCC N stage.

Keywords: endometrioid endometrial cancer, lymph node ratio, log odds of positive lymph nodes, prognosis, SEER 


\section{INTRODUCTION}

Endometrial cancer (EC) was one of the most common gynecological malignancies, with 382,069 new cases expected worldwide in 2018 (1). Histologically, $80 \%$ of the EC patients belonged to type I EC, also known as endometrioid adenocarcinoma. The remaining $20 \%$ of the EC patients were type II EC that was more aggressive in nature (2). The majority of the endometrioid endometrial cancer (EEC) patients were diagnosed at an early stage with a relatively promising prognosis (3). However, the 5-year disease-specific survival (DSS) for node-positive EEC patients ranged from 44 to $77 \%$, which suggested that considerable heterogeneity existed among these patients $(4,5)$. As a result, a more predictive lymph node (LN) staging system was needed for node-positive EEC patients.

It was noted that LN status has been one of the most significant prognostic factors for EEC patients (6). Recently, node-positive EC was divided into N1 (pelvic LN involvement) and N2 (para-aortic LN involvement with or without pelvic LN involvement) (7). This new $\mathrm{N}$ staging principle made sense from the perspective of regional LN drainage, as the pelvic LN basin harbored almost all of the uterine LN drainage while the para-aortic LN involvement might imply further progression $(8,9)$. However, this staging system did not take LN number or ratio into consideration, and previous studies have revealed that a higher number of positive lymph nodes (NPLN) or lymph node ratio (LNR) was related to more dreadful survival for EEC patients (10-12). So, it is necessary to further explore the prognostic significance of these LN schemes.

LNR, defined as NPLN divided by the number of retrieved lymph nodes (NRLN), has been found useful in evaluating the prognosis in node-positive EEC patients $(4,13,14)$. Log odds of positive lymph nodes (LODDS) was a nascent prognostic parameter that has currently been used to assess the prognosis in various malignancies (15-18). To the best of our knowledge, little clinical evidence has recommended the most suitable LN scheme to predict the prognosis for node-positive EEC patients. Meanwhile, the prognostic role of LODDS in EEC patients has not been established in previous studies. Therefore, we conducted this study to compare the predictive performance of six different LN schemes in node-positive EEC patients.

\section{METHODS}

\section{Data Source and Population Selection}

The data were collected from the Surveillance, Epidemiology, and End Results (SEER) database consisting of $\sim 34.6 \%$ of the cancer population in the United States (19). We extracted the relevant data through SEER*Stat software (version 8.4.6; http://seer.cancer.gov/seerstat/) with permission to access the database (Authorization Code: 17548-Nov2020). Since the data available in this program were anonymous, the requirement for informed consent was waived. We created a case listing including all EEC patients (histological codes: 8080/3, 8081/3, 8082/3, 8083/3) diagnosed between 2010 and 2016 based on the third edition of the International Classification of Disease Oncology (ICD-O-3). LN metastasis was defined as macrometastasis or micrometastasis in the sampling $\mathrm{LN}$ according to the pathological findings in the SEER database. Patients who met any of the following criteria would be excluded: (1) Patients with insufficient information regarding LN status, clinicalpathological characteristics, surgical, and follow-up treatment; (2) Patients without LN metastasis (N0 stage); (3) Patients without LN retrieving during the surgery; (4) T4 (bladder mucosa or rectal mucosa was invaded) or M1 (distant metastasis); (5) Patients without hysterectomy; (6) Age $<18$ years; (7) Patients died from surgical complications (survival time $<1$ month); (8) Patients with malignant tumor history. Age at diagnosis was divided into two groups $(<65, \geq 65)$ according to previous studies $(20,21)$.

\section{Number of Retrieved Lymph Nodes, Number of Positive Lymph Nodes, Number of Negative Lymph Nodes, Lymph Node Ratio, and Log Odds of Positive Lymph Nodes Classification}

NRLN represented the total number of retrieved LNs during the surgery. NPLN was defined as the number of positive LNs. Number of negative lymph nodes (NNLN), known as the number of negative LNs among the retrieved LNs, could be estimated as NNLN = NRLN - NPLN. LNR was calculated as NPLN/NRLN. LODDS was defined as $\log _{e}$ [(NPLN $+0.5) /(\mathrm{NNLN}+0.5)]$, where 0.5 was added to both the denominator and numerator to avoid singularity (22). We trichotomized these five continuous variables (NRLN, NPLN, NNLN, LNR, LODDS) via X-tile software (version 3.6.1; Yale University, New Haven, CT, USA) once maximal chi-square value was reached, which was considered to represent the greatest difference in prognosis prediction among the subgroups (23, 24). In our study, NRLN was divided into three subgroups: NRLN1 (1-5), NRLN2 (6-14), NRLN3 ( $\geq 15)$. NPLN was divided into NPLN1 (1), NPLN2 (2-6), and NPLN3 $(\geq 7)$. NNLN was categorized as NNLN1 $(<5)$, NNLN2 (5-13), and NNLN3 $(\geq 14)$. LNR was classified into LNR1 (0.01-0.15), LNR2 (0.16-0.66), and LNR3 $(\geq 0.67)$. LODDS was divided into three subgroups: $-4.07<$ LODDS1 $\leq-1.44,-1.44<$ LODDS2 $\leq 0.49$, and $0.49<$ LODDS $3 \leq 4.32$.

\section{Statistical Analysis}

We conducted a two-step Cox proportional hazard regression analysis to illustrate the association between the different LN schemes and OS. Firstly, univariate Cox regression analysis was performed to select the possible prognostic factors $(p<0.100)$, which would be included in the subsequent multivariate Cox regression analysis. Secondly, we conducted six multivariate Cox regression models with each model incorporating one LN scheme: Model $1(\mathrm{~N})$, Model 2 (NRLN), Model 3 (NPLN), Model 4 (NNLN), Model 5 (LNR), and Model 6 (LODDS). In order to maintain the authenticity of original data, the continuous pattern of the data was processed in the same way. Predictive performance of different LN models was assessed from multiple perspectives. AIC was adopted to evaluate the models' goodness of fit (25). Moreover, C-index was 
TABLE 1 | Clinical and pathological characteristics and survival analysis of patients.

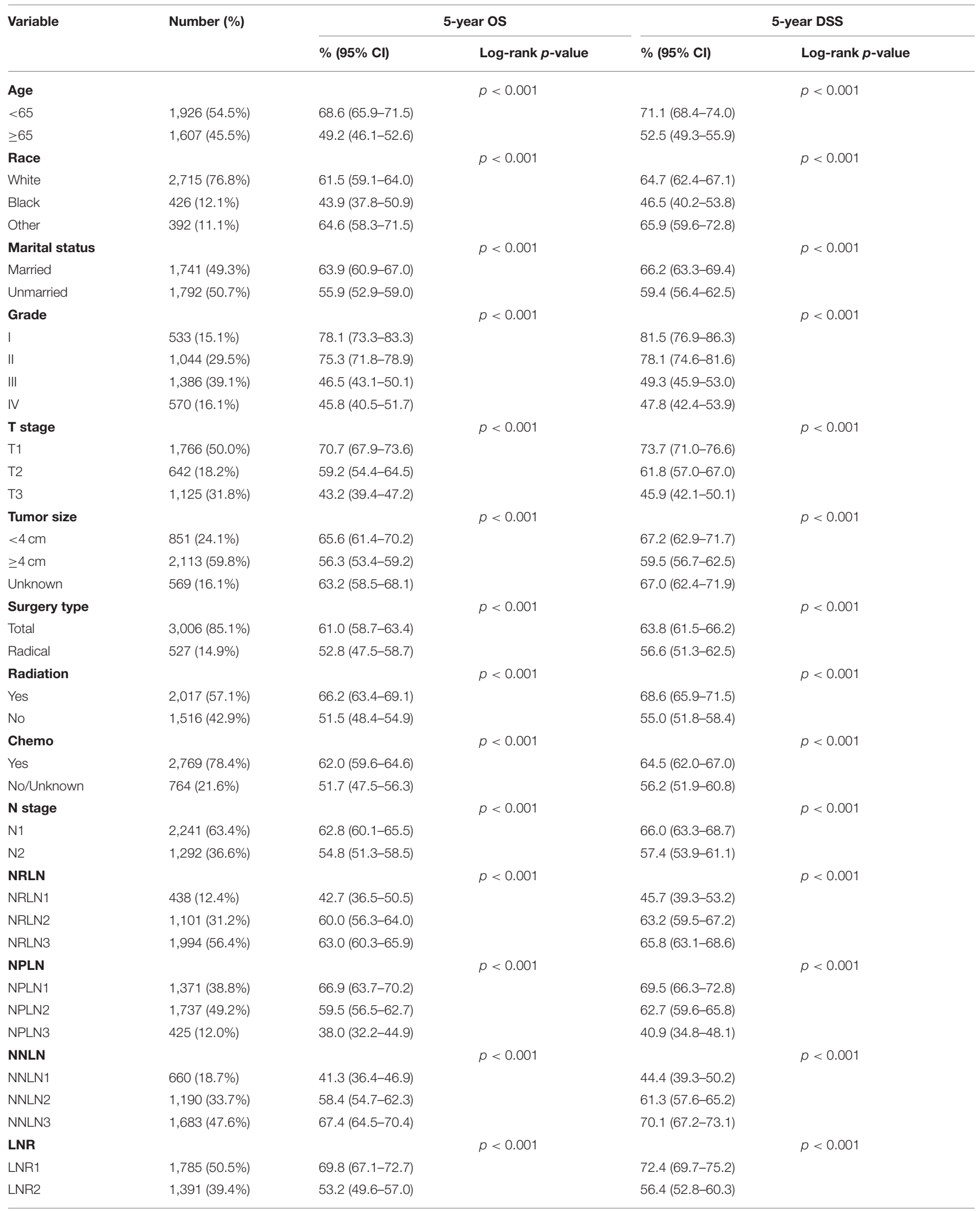


TABLE 1 | Continued

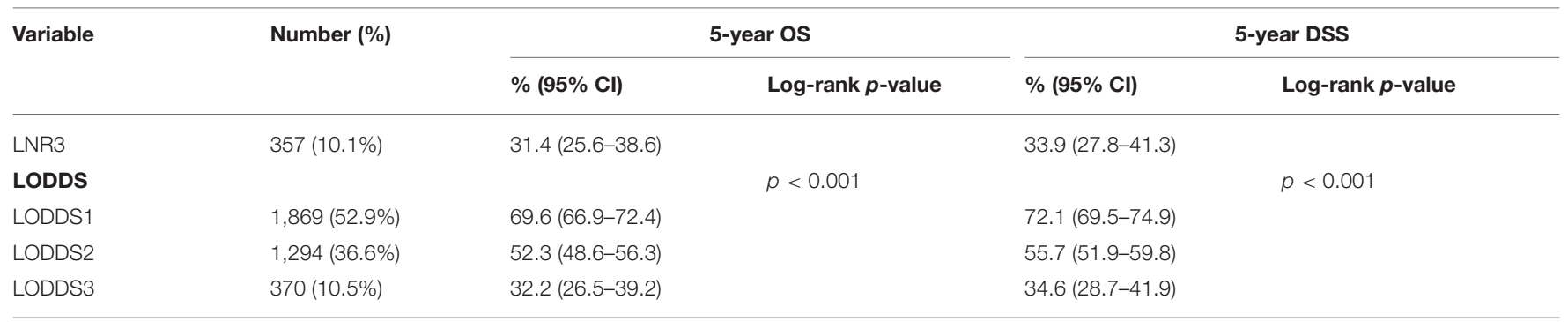

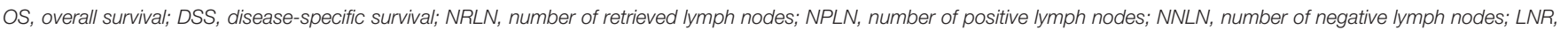
lymph node ratio; LODDS, log odds of positive lymph nodes; $\mathrm{Cl}$, confidence interval.

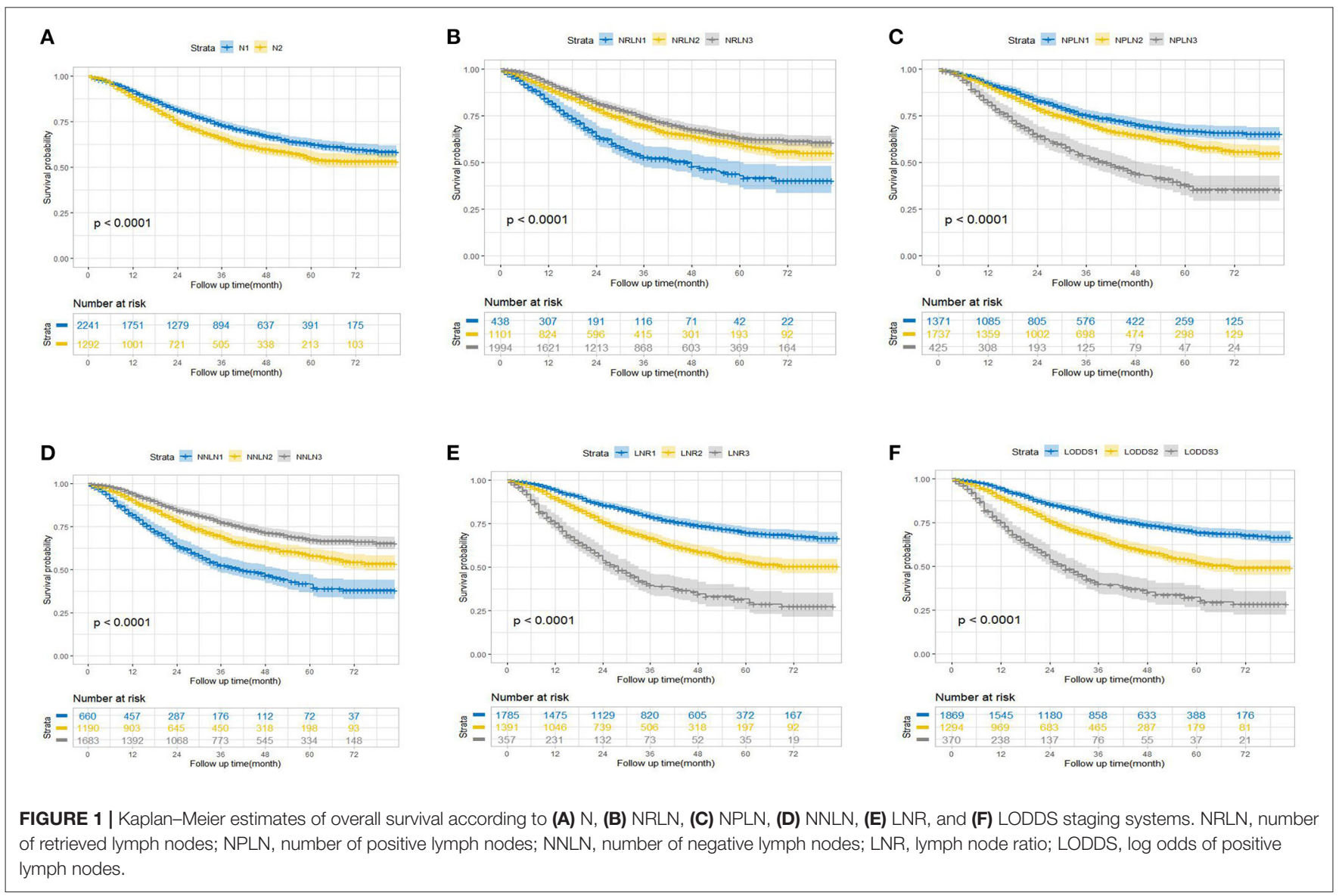

calculated to appraise the discrimination power of the LN models (26).

The correlations between the continuous patterns of NRLN, NPLN, NNLN, LNR, and LODDS were visualized and compared with scatter plot and Pearson correlation coefficient (r), respectively. Sensitivity analysis using DSS as endpoint was then performed. All analyses were performed using $\mathrm{R}$ software (version 3.6.1; http://www.r-project.org) and IBM SPSS 22.0 (Armonk, NY). A two-tailed $p<0.05$ is recognized as statistically significant.

\section{RESULTS}

\section{Patient Characteristics and Survival Analysis}

A total of 3,533 EEC patients remained in our final study cohort. The selection process was shown in detail (Supplementary Table 1). We summarized the baseline clinicalpathological and demographic characteristics in Table 1. The median NRLN was 16 [interquartile range (IQR): 9-25], and mean NRLN was $18.47 \pm 12.57$. Similarly, the median NPLN and mean NPLN were 2 (IQR: $1-4$ ) and $3.49 \pm 4.68$, respectively. The 
mean NNLN was $14.98 \pm 11.67$, and the median NNLN was 13 (IQR: 6-21). The mean LNR and median LNR were 0.15 (IQR: $0.08-0.33)$ and $0.26 \pm 0.26$. The mean LODDS was $-1.29 \pm$ 1.26 , and the median LODDS was -1.53 (IQR: -2.20 to -0.59 ). More than half of the NRLN in eligible patients exceeded 15, which further ensured the accuracy of LN sampling.

The survival probability for node-positive EEC patients increased as the increment of the value of NRLN and NNLN. For example, the 5-year OS increased from $42.7 \%$ (36.5-50.5\%) for NRLN1 to $63.0 \%(60.3-65.9 \%)$ for NRLN3. Furthermore, this also can be validated by the restricted cubic spline analysis that mortality risk decreased as NRLN increased (Supplementary Figure 1A). However, the survival probability for node-positive EEC patients decreased as the increment of NPLN, LNR, and LODDS. For instance, the 5year DSS was reduced from $72.1 \%(69.5-74.9 \%)$ for LODDS1 to $34.6 \%(28.7-41.9 \%)$ for LODDS3 $(P<0.001)$. Still, as shown in Supplementary Figure 1E, mortality risk increased as LODDS increased. The Kaplan-Meier curves for OS and DSS stratified by different LN schemes were presented in Figure 1 and Supplementary Figure 2, respectively. Six multivariate Cox models were set up, with each model incorporating one LN scheme and other prognostic factors identified in the univariate Cox regression analysis (Supplementary Table 2), which were exhibited as Model $1(\mathrm{~N})$, Model 2 (NRLN), Model 3 (NPLN), Model 4 (NNLN), Model 5 (LNR), and Model 6 (LODDS) in Table 2. We found that $\mathrm{N}$ stage $(\mathrm{N} 1 / \mathrm{N} 2)$ was not significantly associated with survival outcomes regardless of DSS $(p=0.253)$ or OS $(p=0.145)$.

\section{Prognostic Performance Among Number of Retrieved Lymph Nodes, Number of Positive Lymph Nodes, Number of Negative Lymph Nodes, Lymph Node Ratio, and Log Odds of Positive Lymph Nodes}

We excluded N stage (N1/N2) from the comparison of prognostic performance seeing that $\mathrm{N}$ stage did not show any prognostic ability in Model 1. The predictive performance of the remaining five Cox models was assessed (Table 3). Model 5 (LNR) showed the lowest AIC (AIC: 14,453.81) and the highest C-index value (C-index: 0.735) among the five models in categorical pattern. Subsequently, we compared these five models in continuous pattern, which revealed that Model 5 (LNR) still had the lowest AIC (AIC: 14,456.17), but the highest C-index value was obtained by Model 6 (LODDS) (C-index: 0.736). Interestingly, we observed LODDS and LNR had almost identical C-index values in OS regardless of continuous (LODDS vs. LNR: 0.736 vs. 0.735 ) or categorical pattern (LODDS vs. LNR: 0.734 vs. 0.735). Therefore, it would be reckless to simply conclude LNR or LODDS as the best-performance model in predicting OS for EEC patients. Meanwhile, the C-index value for all the five models exceeded 0.70 , which demonstrated a considerably good predictive accuracy (27).

\section{Correlation Among Different Lymph Node Schemes}

Scatter plot was used to visualize the relationship among different LN schemes, which demonstrated that LNR was more correlated with LODDS than other LN schemes $(r=0.913$ vs. $r=0.148, r=0.181$ and $r=0.341$; all $p<0.001)$. Besides, LNR and LODDS were positively correlated with NPLN (Figures 2B,F); however, they were negatively correlated with NRLN (Figures 2A,E) and NNLN (Figures 2C,G). Notably, LNR and LODDS differentiate the prognosis for patients with the same NPLN even when NPLN $\leq 5$ (Figures 2B,F). Similarly, LNR and LODDS could remain heterogeneous even when NRLN is small (Figures 2A,E). In addition, LNR was positively associated with LODDS, though the correlation between them was not precisely linear (Figures 2D,H). As shown in Figure 2D, the curve became much steeper when LNR $\leq 0.2$ or LNR $\geq 0.8$, which suggested that LNR increased much slower than LODDS in this range. Therefore, LODDS might perform better in predicting prognosis when LNR was either low or high.

\section{Sensitivity Analysis}

We used the same approach to process DSS. Multivariate Cox analysis revealed that Model $1(\mathrm{~N})$ was not an independent risk factor for DSS either (Supplementary Table 3). In the assessment of predictive models, Model 5 (LNR) remained the best performer in the evaluation of goodness of fit, which had the lowest AIC in both continuous (AIC: 12,866.34) and categorical patterns (AIC: 12,863.51; Table 3). Similarly, LNR and LODDS still had the resembling discrimination power regardless of continuous pattern (C-index: LNR vs. LODDS = 0.738 vs. 0.739 ) or categorical pattern (C-index: LNR vs. LODDS $=0.737$ vs. 0.737 ; Table 3 ).

\section{DISCUSSION}

The updated $\mathrm{N}$ staging system based on anatomic location was considered the optimal way to predict the prognosis for nodepositive EC patients currently, but this LN scheme mingled EEC patients with non-EEC patients, which overlooked the fact that EEC patients had more promising outcomes than nonEEC patients (28). In our study, the American Joint Committee on Cancer (AJCC) $\mathrm{N}$ stage was meaningless in prognosis prediction for node-positive EEC patients after surgery, which was consistent with the previous study showing that EEC patients with isolated pelvic LN metastasis shared a similar prognosis with EEC patients with isolated para-aortic LN metastasis (29).

In terms of NRLN, previous study showed that more than 20 retrieved LNs during the surgery could benefit the survival of EEC patients; however, this study included both the nodepositive and node-negative EEC patients (30). In our study, the optimal NRLN was 15, and increasing NRLN was linked to better DSS and OS, which deserved to be further evaluated. We have noticed that the NPLN scheme has been used to assess prognosis for EEC patients, which revealed that patients with only one LN involvement outperformed patients with multiple 
TABLE 2 | Multivariable Cox regression analysis (Models 1-6) of prognostic predictors for OS.

\begin{tabular}{|c|c|c|c|c|c|c|c|c|c|c|c|c|}
\hline \multirow[b]{2}{*}{ Variable } & \multicolumn{2}{|c|}{ Model 1 (N) } & \multicolumn{2}{|c|}{ Model 2 (NRLN) } & \multicolumn{2}{|c|}{ Model 3 (NPLN) } & \multicolumn{2}{|c|}{ Model 4 (NNLN) } & \multicolumn{2}{|c|}{ Model 5 (LNR) } & \multicolumn{2}{|c|}{ Model 6 (LODDS) } \\
\hline & HR $(95 \%$ Cl) & $p$-value & HR (95\% Cl) & $p$-value & HR $(95 \% \mathrm{Cl})$ & $p$-value & HR $(95 \% \mathrm{Cl})$ & $p$-value & HR $(95 \%$ ci) & $p$-value & HR $(95 \% \mathrm{Cl})$ & $p$-value \\
\hline \multicolumn{13}{|l|}{ Age } \\
\hline$<65$ & 1 & & 1 & & 1 & & 1 & & 1 & & 1 & \\
\hline$\geq 65$ & $\begin{array}{c}1.651(1.450 \\
1.879)\end{array}$ & $p<0.001$ & $\begin{array}{c}1.670(1.467 \\
1.901)\end{array}$ & $p<0.001$ & $\begin{array}{c}1.648(1.447 \\
1.876)\end{array}$ & $p<0.001$ & $\begin{array}{c}1.671(1.467 \\
1.903)\end{array}$ & $p<0.001$ & $\begin{array}{c}1.684(1.479 \\
1.918)\end{array}$ & $p<0.001$ & $\begin{array}{c}1.680(1.475 \\
1.913)\end{array}$ & $p<0.001$ \\
\hline \multicolumn{13}{|l|}{ Race } \\
\hline White & 1 & & 1 & & 1 & & 1 & & 1 & & 1 & \\
\hline Black & $\begin{array}{c}1.219(1.023 \\
1.453)\end{array}$ & $p=0.027$ & $\begin{array}{c}1.210(1.015 \\
1.442)\end{array}$ & $p=0.034$ & $\begin{array}{c}1.191 \text { (0.999, } \\
1.419)\end{array}$ & $p=0.052$ & $\begin{array}{c}1.154(0.968 \\
1.377)\end{array}$ & $p=0.111$ & $\begin{array}{c}1.122(0.940 \\
1.339)\end{array}$ & $p=0.201$ & $\begin{array}{c}1.125(0.943 \\
1.342)\end{array}$ & $p=0.192$ \\
\hline Other & $\begin{array}{c}0.864(0.692 \\
1.080)\end{array}$ & $p=0.199$ & $\begin{array}{c}0.858 \text { (0.687 } \\
1.072)\end{array}$ & $p=0.177$ & $\begin{array}{c}0.881(0.705 \\
1.101)\end{array}$ & $p=0.264$ & $\begin{array}{c}0.842(0.674 \\
1.052)\end{array}$ & $p=0.130$ & $\begin{array}{c}0.840(0.672 \\
1.050)\end{array}$ & $p=0.105$ & $\begin{array}{c}0.841(0.678 \\
1.051)\end{array}$ & $p=0.127$ \\
\hline \multicolumn{13}{|c|}{ Marital status } \\
\hline Married & 1 & & 1 & & 1 & & 1 & & 1 & & 1 & \\
\hline Unmarried & $\begin{array}{c}1.106(0.971 \\
1.258)\end{array}$ & $p=0.129$ & $\begin{array}{c}1.090(0.958 \\
1.241)\end{array}$ & $p=0.191$ & $\begin{array}{c}1.109 \text { (0.974 } \\
1.262)\end{array}$ & $p=0.118$ & $\begin{array}{c}1.088(0.955 \\
1.238)\end{array}$ & $p=0.204$ & $\begin{array}{c}1.107(0.973 \\
1.260)\end{array}$ & $p=0.123$ & $\begin{array}{c}1.100(0.966 \\
1.252)\end{array}$ & $p=0.151$ \\
\hline \multicolumn{13}{|l|}{ Grade } \\
\hline I & 1 & & 1 & & 1 & & 1 & & 1 & & 1 & \\
\hline$\|$ & $\begin{array}{c}1.218(0.925 \\
1.603)\end{array}$ & $p=0.160$ & $\begin{array}{c}1.237 \text { (0.939, } \\
1.629)\end{array}$ & $p=0.130$ & $\begin{array}{c}1.197(0.909 \\
1.576)\end{array}$ & $p=0.200$ & $\begin{array}{c}1.239 \text { (0.941 } \\
1.631)\end{array}$ & $p=0.127$ & $\begin{array}{c}1.228(0.933 \\
1.617)\end{array}$ & $p=0.142$ & $\begin{array}{c}1.210(0.919 \\
1.592)\end{array}$ & $p=0.175$ \\
\hline III & $\begin{array}{c}2.702(2.101 \\
\quad 3.475)\end{array}$ & $p<0.001$ & $\begin{array}{c}2.705(2.103 \\
3.479)\end{array}$ & $p<0.001$ & $\begin{array}{c}2.615(2.032 \\
3.365)\end{array}$ & $p<0.001$ & $\begin{array}{c}2.666(2.072 \\
3.428)\end{array}$ & $p<0.001$ & $\begin{array}{c}2.581(2.006 \\
3.321)\end{array}$ & $p<0.001$ & $\begin{array}{c}2.556(1.986 \\
3.289)\end{array}$ & $p<0.001$ \\
\hline IV & $\begin{array}{c}2.880(2.190 \\
3.787)\end{array}$ & $p<0.001$ & $\begin{array}{c}2.958(2.249, \\
3.891)\end{array}$ & $p<0.001$ & $\begin{array}{c}2.781(2.114 \\
3.659)\end{array}$ & $p<0.001$ & $\begin{array}{c}2.934(2.231 \\
3.858)\end{array}$ & $p<0.001$ & $\begin{array}{c}2.866(2.180 \\
3.769)\end{array}$ & $p<0.001$ & $\begin{array}{c}2.834(2.155 \\
3.726)\end{array}$ & $p<0.001$ \\
\hline \multicolumn{13}{|l|}{ T stage } \\
\hline $\mathrm{T} 1$ & 1 & & 1 & & 1 & & 1 & & 1 & & 1 & \\
\hline T2 & $\begin{array}{c}1.262(1.052 \\
1.513)\end{array}$ & $p=0.012$ & $\begin{array}{c}1.257(1.048 \\
1.507)\end{array}$ & $p=0.014$ & $\begin{array}{c}1.249 \text { (1.041 } \\
1.499)\end{array}$ & $p=0.017$ & $\begin{array}{c}1.225(1.022 \\
1.470)\end{array}$ & $p=0.028$ & $\begin{array}{c}1.217(1.015 \\
1.460)\end{array}$ & $p=0.034$ & $\begin{array}{c}1.222(1.019 \\
1.465)\end{array}$ & $p=0.031$ \\
\hline T3 & $\begin{array}{c}2.127(1.837 \\
2.464)\end{array}$ & $p<0.001$ & $\begin{array}{c}2.058(1.777 \\
2.384)\end{array}$ & $p<0.001$ & $\begin{array}{c}2.015 \text { (1.736, } \\
2.338)\end{array}$ & $p<0.001$ & $\begin{array}{c}1.950(1.682 \\
2.262)\end{array}$ & $p<0.001$ & $\begin{array}{c}1.798(1.546 \\
2.091)\end{array}$ & $p<0.001$ & $\begin{array}{c}1.796(1.544 \\
2.089)\end{array}$ & $p<0.001$ \\
\hline \multicolumn{13}{|c|}{ Tumor size } \\
\hline$<4 \mathrm{~cm}$ & 1 & & 1 & & 1 & & 1 & & 1 & & 1 & \\
\hline$\geq 4 \mathrm{~cm}$ & $\begin{array}{c}1.237(1.051 \\
1.456)\end{array}$ & $p=0.011$ & $\begin{array}{c}1.224(1.039 \\
1.441)\end{array}$ & $p=0.015$ & $\begin{array}{c}1.218(1.034 \\
1.434)\end{array}$ & $p=0.018$ & $\begin{array}{c}1.207(1.025 \\
1.422)\end{array}$ & $p=0.024$ & $\begin{array}{c}1.216(1.033 \\
1.432)\end{array}$ & $p=0.019$ & $\begin{array}{c}1.213(1.030 \\
1.428)\end{array}$ & $p=0.021$ \\
\hline Unknown & $\begin{array}{c}1.063(0.862 \\
1.311)\end{array}$ & $p=0.567$ & $\begin{array}{c}1.075(0.872 \\
1.325)\end{array}$ & $p=0.498$ & $\begin{array}{c}1.042(0.845 \\
1.285)\end{array}$ & $p=0.701$ & $\begin{array}{c}1.063(0.862 \\
1.311)\end{array}$ & $p=0.564$ & $\begin{array}{c}1.020(0.827 \\
1.258)\end{array}$ & $p=0.855$ & $\begin{array}{c}1.025 \text { (0.831 } \\
1.264)\end{array}$ & $p=0.820$ \\
\hline \multicolumn{13}{|c|}{ Surgery type } \\
\hline Total & 1 & & 1 & & 1 & & 1 & & 1 & & 1 & \\
\hline Radical & $\begin{array}{c}1.189(1.011 \\
1.399)\end{array}$ & $p=0.036$ & $\begin{array}{c}1.215(1.033 \\
1.430)\end{array}$ & $p=0.019$ & $\begin{array}{c}1.182(1.005 \\
1.391)\end{array}$ & $p=0.043$ & $\begin{array}{c}1.228(1.043 \\
1.445)\end{array}$ & $p=0.014$ & $\begin{array}{c}1.204(1.024 \\
1.417)\end{array}$ & $p=0.025$ & $\begin{array}{c}1.208(1.027 \\
1.422)\end{array}$ & $p=0.023$ \\
\hline
\end{tabular}


TABLE 2 | Continued

\begin{tabular}{|c|c|c|c|c|c|c|c|c|c|c|c|c|}
\hline \multirow[b]{2}{*}{ Variable } & \multicolumn{2}{|c|}{ Model 1 (N) } & \multicolumn{2}{|c|}{ Model 2 (NRLN) } & \multicolumn{2}{|c|}{ Model 3 (NPLN) } & \multicolumn{2}{|c|}{ Model 4 (NNLN) } & \multicolumn{2}{|c|}{ Model 5 (LNR) } & \multicolumn{2}{|c|}{ Model 6 (LODDS) } \\
\hline & HR $(95 \% \mathrm{Cl})$ & $p$-value & HR (95\% Cl) & $p$-value & HR $(95 \% \mathrm{Cl})$ & $p$-value & HR $(95 \% \mathrm{Cl})$ & $p$-value & HR (95\% ci) & $p$-value & HR $(95 \% \mathrm{Cl})$ & $p$-value \\
\hline \multicolumn{13}{|l|}{ Radiation } \\
\hline Yes & 1 & & 1 & & 1 & & 1 & & 1 & & 1 & \\
\hline No & $\begin{array}{c}1.567(1.373 \\
1.787)\end{array}$ & $p<0.001$ & $\begin{array}{c}1.550(1.359 \\
1.767)\end{array}$ & $p<0.001$ & $\begin{array}{c}1.545(1.354 \\
1.763)\end{array}$ & $p<0.001$ & $\begin{array}{c}1.553(1.361 \\
1.770)\end{array}$ & $p<0.001$ & $\begin{array}{c}1.505(1.319 \\
1.717)\end{array}$ & $p<0.001$ & $\begin{array}{c}1.511(1.324 \\
1.724)\end{array}$ & $p<0.001$ \\
\hline \multicolumn{13}{|l|}{ Chemo } \\
\hline Yes & 1 & & 1 & & 1 & & 1 & & 1 & & 1 & \\
\hline No/Unknown & $\begin{array}{c}1.489(1.287 \\
1.722)\end{array}$ & $p<0.001$ & $\begin{array}{c}1.475(1.276 \\
1.706)\end{array}$ & $p<0.001$ & $\begin{array}{c}1.504(1.300 \\
1.741)\end{array}$ & $p<0.001$ & $\begin{array}{c}1.462(1.264 \\
1.691)\end{array}$ & $p<0.001$ & $\begin{array}{c}1.506(1.302 \\
1.742)\end{array}$ & $p<0.001$ & $\begin{array}{c}1.502(1.298 \\
1.737)\end{array}$ & $p<0.001$ \\
\hline \multicolumn{13}{|l|}{ N stage } \\
\hline N1 & 1 & & & & & & & & & & & \\
\hline N2 & $\begin{array}{c}1.078(0.948 \\
1.226)\end{array}$ & $p=0.253$ & & & & & & & & & & \\
\hline \multicolumn{13}{|l|}{ NRLN } \\
\hline NRLN1 & & & 1 & & & & & & & & & \\
\hline NRLN2 & & & $\begin{array}{c}0.667(0.552 \\
0.806)\end{array}$ & $p<0.001$ & & & & & & & & \\
\hline NRLN3 & & & $\begin{array}{c}0.590(0.494 \\
0.703)\end{array}$ & $p<0.001$ & & & & & & & & \\
\hline \multicolumn{13}{|l|}{ NPLN } \\
\hline NPLN1 & & & & & 1 & & & & & & & \\
\hline NPLN2 & & & & & $\begin{array}{c}1.135(0.983 \\
1.311)\end{array}$ & $p=0.083$ & & & & & & \\
\hline NPLN3 & & & & & $\begin{array}{c}1.595(1.321 \\
1.926)\end{array}$ & $p<0.001$ & & & & & & \\
\hline \multicolumn{13}{|l|}{ NNLN } \\
\hline NNLN1 & & & & & & & 1 & & & & & \\
\hline NNLN2 & & & & & & & $\begin{array}{c}0.643(0.547 \\
0.756)\end{array}$ & $p<0.001$ & & & & \\
\hline NNLN3 & & & & & & & $\begin{array}{c}0.507(0.432 \\
0.596)\end{array}$ & $p<0.001$ & & & & \\
\hline \multicolumn{13}{|l|}{ LNR } \\
\hline LNR1 & & & & & & & & & 1 & & & \\
\hline LNR2 & & & & & & & & & $\begin{array}{c}1.490(1.294 \\
1.716)\end{array}$ & $p<0.001$ & & \\
\hline LNR3 & & & & & & & & & $\begin{array}{c}2.624(2.172 \\
3.169)\end{array}$ & $p<0.001$ & & \\
\hline \multicolumn{13}{|l|}{ LODDS } \\
\hline LODDS1 & & & & & & & & & & & 1 & \\
\hline LODDS2 & & & & & & & & & & & $\begin{array}{c}1.492(1.296 \\
1.719)\end{array}$ & $p<0.001$ \\
\hline LODDS3 & & & & & & & & & & & $\begin{array}{c}2.566(2.131 \\
3.090)\end{array}$ & $p<0.001$ \\
\hline
\end{tabular}

OS, overall survival; NRLN, number of retrieved lymph nodes; NPLN, number of positive lymph nodes; NNLN, number of negative lymph nodes; LNR, Iymph node ratio; LODDS, Iog odds of positive lymph nodes; Cl, confidence interval. 
TABLE 3 | Predictive performance of different LN schemes.

\begin{tabular}{|c|c|c|c|c|}
\hline Model & \multicolumn{2}{|c|}{ os } & \multicolumn{2}{|c|}{ DSS } \\
\hline Model 2 (NRLN) & $14,528.27$ & $0.723(0.707-0.739)$ & $12,935.51$ & $0.725(0.709-0.741)$ \\
\hline Model 3 (NPLN) & $14,536.77$ & $0.724(0.709-0.740)$ & $12,940.20$ & $0.728(0.712-0.743)$ \\
\hline Model 4 (NNLN) & $14,488.16$ & $0.729(0.714-0.745)$ & $12,898.99$ & $0.731(0.715-0.746)$ \\
\hline Model 6 (LODDS) & $14,453.98$ & $0.734(0.718-0.750)$ & $12,864.37$ & $0.737(0.721-0.753)$ \\
\hline \multicolumn{5}{|l|}{ Continuous } \\
\hline Model 2 (NRLN) & $14,537.68$ & $0.722(0.706-0.737)$ & $12,941.22$ & $0.724(0.708-0.739)$ \\
\hline Model 3 (NPLN) & $14,548.50$ & $0.733(0.718-0.749)$ & $12,951.54$ & $0.726(0.710-0.742)$ \\
\hline Model 4 (NNLN) & $14,508.62$ & $0.726(0.710-0.741)$ & $12,914.70$ & $0.728(0.712-0.743)$ \\
\hline
\end{tabular}

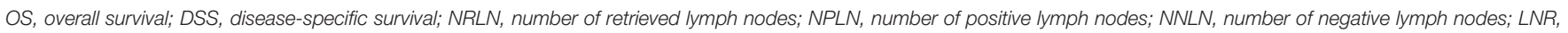
lymph node ratio; LODDS, log odds of positive lymph nodes; Cl, confidence interval; AIC, Akaike information criterion.

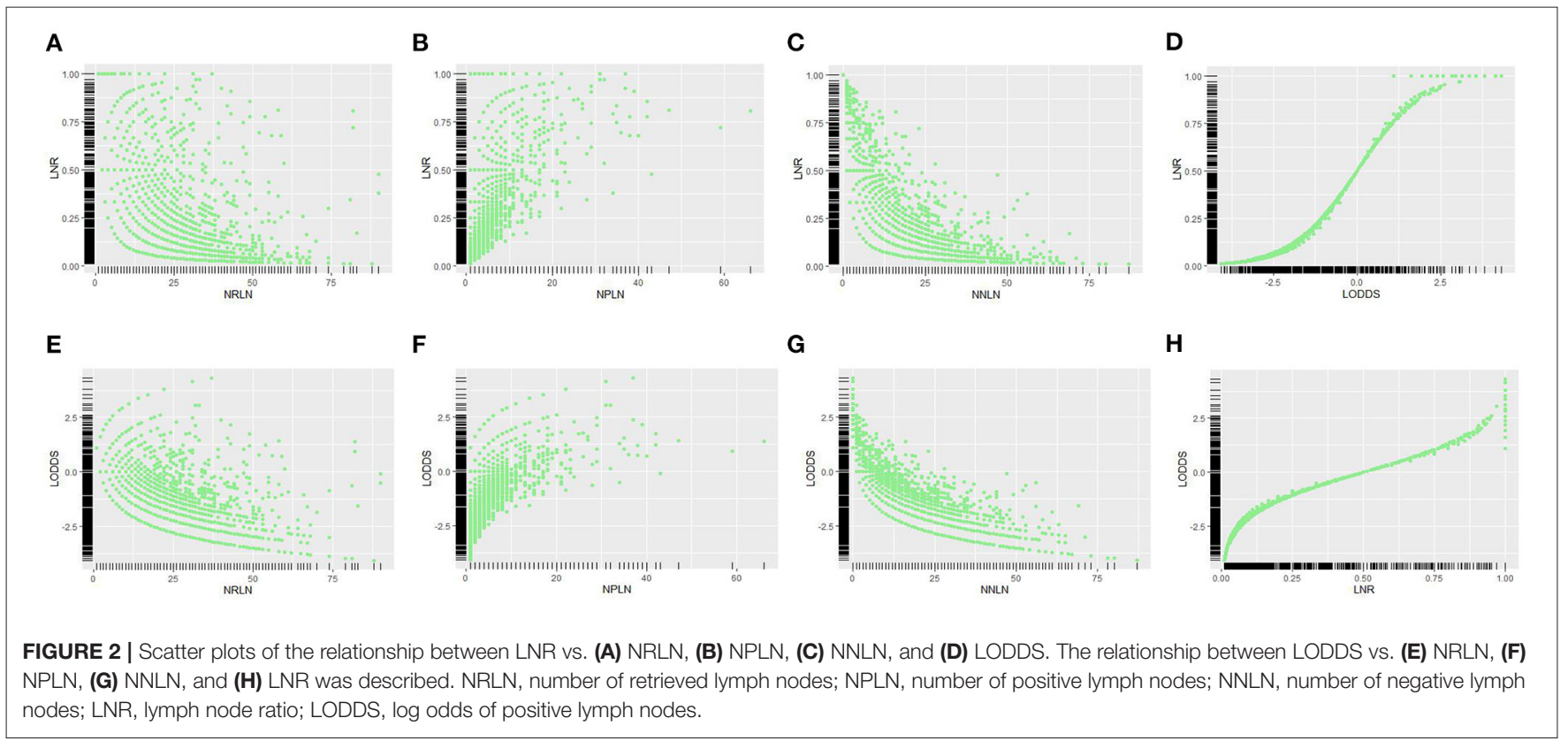

LN involvement regarding OS and DSS (12). However, not only the NPLN scheme could be affected by NRLN during the surgery but also the pathological examination procedure might have an impact on the result, which could lead to miscounting of NPLN. Similarly, the NNLN scheme shared the same concerns with NPLN (18).

The LNR scheme covered the information of both NPLN and NRLN, which was able to surmount the limitations of the above number-based LN schemes theoretically. Numerous studies had established the prognostic role of LNR scheme in other gynecological oncologies $(18,31,32)$. In EC patients, the LNR scheme was deemed to be a novel independent prognostic index after surgery $(4,10,13)$. However, this scheme still had its limitations. Firstly, LNR scheme was genetically not suitable for distinguishing the survival among node-negative EEC patients. Secondly, patients with different NRLN and NPLN might share identical LNRs, which brought up the obvious question on whether EEC patients with one positive LN out of one retrieved LN performed equally in OS and DDS compared to patients with five positive LNs out of five retrieved LNs. As a nascent parameter, LODDS scheme could make up for LNR, which has been thoroughly investigated in various malignancies and showed superior prognostic predictive power than the LNR scheme (15-17, 24, 33, 34).

$\mathrm{N}$ staging (Model 1) was washed out for not being an independent prognostic factor for survival, which suggested 
that more aggressive adjuvant therapy for N2 patients than N1 patients was debatable. In the current study, node-positive EEC patients with more NRLN, more NNLN, less NPLN, lower LNR, and lower LODDS performed better in DSS and OS (Table 2, Supplementary Table 3), which implied that more aggressive adjuvant therapy and closer follow-up might be arranged for these patients. Scatter plots were conducted to visualize the reciprocal correlations among these LN schemes, which revealed the tightest relationship between LNR and LODDS (Figures 2D,H). This was not hard to explain given that these two schemes were both ratio-based LN schemes.

Some strengths and limitations of this study should be noted. High-volume data from the SEER registry ensured the sophisticated generalizability and universality of this study. On the other hand, each multivariate Cox regression model was assessed not just in discrimination power but also in goodness of fit, which resulted in the addition of comprehensiveness and rigorousness to this study. Moreover, we appraised the predictive power of these models in continuous and categorical patterns to avoid data distortion. Last but not least, ratio-based LN schemes were demonstrated to be more useful in prognosis prediction for node-positive EEC patients, which provided an alternative way to assess the survival outcomes for these patients. However, several limitations in our study also needed to be noted. Firstly, as a retrospective study, selection bias was difficult to avoid, and prospective studies are welcome in the future. Secondly, some detailed information as to whether sentinel node mapping or systemic lymphadenectomy was used during the surgery and whether para-aortic LN sampling was performed had not been included in the SEER database, which might impact the assessment of NRLN. Since ratio-based LN schemes generally performed better than did number-based LN schemes, we believe that this would not significantly affect our results. Surely, more precise evaluations about LN schemes for node-positive EEC patients are welcome. Finally, the LNR ranged from 0 to 1 , and the LODDS varied from negative infinity to positive infinity theoretically, but in the current study, we were confined to the setting of $-4.07<$ LODDS $\leq 4.32$ and $0.01 \leq \mathrm{LNR} \leq 1$. As a result, it remained unclear whether the values exceeding these ranges had the same predictive performance.

\section{REFERENCES}

1. World Health Organization, International Agency for Research on Cancer. Cancer Today 2018 Statistics. Available online at: https:/gco.iarc.fr/today/ home

2. Wright JD, Barrena Mendel NI, Sehouli J, Fujiwaka K, Herzog TJ. Contemporary management of endometrial cancer. Obstetr Gynecol Int. (2012) 379:1352-60. doi: 10.1016/S0140-6736(12) 60442-5

3. Bakkum-Gamez JN, Gonzalez-Bosquet J, Laack NN, Mariani A, Dowdy SC. Current issues in the management of endometrial cancer. Mayo Clin Proc. (2008) 83:97-112. doi: 10.4065/83.1.97

4. Chan JK, Kapp DS, Cheung MK, Osann K, Shin JY, Cohn D, et al. The impact of the absolute number and ratio of positive lymph nodes on survival of endometrioid uterine cancer patients. Br J Cancer. (2007) 97:60511. doi: $10.1038 /$ sj.bjc. 6603898

\section{CONCLUSION}

The AJCC $\mathrm{N}$ staging system (N1/N2) could not differentiate the prognosis for node-positive EEC patients in our study, which might suggest that a more aggressive adjuvant therapy was not necessary for N2 EEC patients than N1 patients. Moreover, we firstly established the prognostic role of the LODDS for node-positive EEC patients. LODDS had superior discrimination power than other LN schemes in continuous pattern, but LNR performed best in categorical patternboth of which should be more significant in predicting the survival outcomes for node-positive EEC patients than AJCC $\mathrm{N}$ stage.

\section{DATA AVAILABILITY STATEMENT}

The datasets presented in this study can be found in online repositories. The names of the repository/repositories and accession number(s) can be found at: SEER.

\section{AUTHOR CONTRIBUTIONS}

X-LY: conception or design and manuscript writing. X-LY and M-MW: provision of study material or patients. X-LY and $\mathrm{NH}$ : collection and/or assembly of data. X-LY, NH, and M-MW: data analysis and interpretation. HL and D-JW: manuscript revision. All authors contributed to the article and approved the submitted version.

\section{ACKNOWLEDGMENTS}

We would like to thank those who have been involved in the establishment of the SEER database and making this database available online.

\section{SUPPLEMENTARY MATERIAL}

The Supplementary Material for this article can be found online at: https://www.frontiersin.org/articles/10.3389/fmed. 2021.688535/full\#supplementary-material

5. Turan T, Ureyen I, Duzguner I, Ozkaya E, Tasci T, karalok A, et al. Analysis of patients with stage IIIC endometrial cancer. Int J Gynecol Cancer. (2014) 24:1033-41. doi: 10.1097/IGC.0000000000000154

6. Mariani A, Webb MJ, Keeney GL, Aletti G, Podratz KC. Predictors of lymphatic failure in endometrial cancer. Gynecol Oncol. (2002) 84:43742. doi: 10.1006/gyno.2001.6550

7. Amant F, Mirza MR, Koskas M, Creutzberg CL. Cancer of the corpus uteri. Int J Gynaecol Obstet. (2018) 143(Suppl. 2):37-50. doi: 10.1002/ijgo.12612

8. Kumar S, Podratz KC, Bakkum-Gamez JN, Dowdy SC, Weaver AL, McGree $\mathrm{ME}$, et al. Prospective assessment of the prevalence of pelvic, paraaortic and high paraaortic lymph node metastasis in endometrial cancer. Gynecol Oncol. (2014) 132:38-43. doi: 10.1016/j.ygyno.2013.10.002

9. James JA, Rakowski JA, Jeppson CN, Stavitzski NM, Ahmad S, Holloway RW. Robotic transperitoneal infra-renal aortic lymphadenectomy in early-stage endometrial cancer. Gynecol Oncol. (2015) 136:285-92. doi: 10.1016/j.ygyno.2014.12.028 
10. Buldukoglu OC, Turker A, Usubutun A, Salman MC. Relationship of lymph node status with survival and recurrence among women with endometrial cancer. Int J Gynaecol Obstet. (2020) 151:267-71. doi: 10.1002/ijgo.13309

11. Ayhan A, Topfedaisi Ozkan N, Öz M, Kimyon Comert G, Firat Cuylan Z, Çoban G, et al. Impact of lymph node ratio on survival in stage IIIC endometrioid endometrial cancer: a Turkish Gynecologic Oncology Group study. J Gynecol Oncol. (2018) 29:e48. doi: 10.3802/jgo.2018.29.e48

12. Stefano U, Francesca F, Stefano G, Francesco F, Pierandrea DI, Giacomo C, et al. Survival in clinical stage I endometrial cancer with single vs. multiple positive pelvic nodes: results of a multi-institutional Italian study. J Gynecol Oncol. (2018) 29:e100. doi: 10.3802/jgo.2018.29.e100

13. Fleming ND, Soliman PT, Westin SN, dos Reis R, Munsell M, Klopp AH, et al. Impact of lymph node ratio and adjuvant therapy in node-positive endometrioid endometrial cancer. Int J Gynecol Cancer. (2015) 25:143744. doi: 10.1097/IGC.0000000000000510

14. Polterauer S, Khalil S, Zivanovic O, Abu-Rustum NR, Hofstetter G, Concin N, et al. Prognostic value of lymph node ratio and clinicopathologic parameters in patients diagnosed with stage IIIC endometrial cancer. Obstet Gynecol. (2012) 119:1210-8. doi: 10.1097/AOG.0b013e318255060c

15. Yu Y, Zhang P, Yao RQ, Wang J, Wang P, Xue X, et al. Prognostic value of log odds of positive lymph nodes in node-positive lung squamous cell carcinoma patients after surgery: a SEER population-based study. Transl Lung Cancer Res. (2020) 9:1285-301. doi: 10.21037/tlcr-20-193

16. Scarinci A, Di Cesare T, Cavaniglia D, Tiziano N, Michelle C, Giulia C, et al. The impact of log odds of positive lymph nodes (LODDS) in colon and rectal cancer patient stratifification: a single-center analysis of 323 patients. Updates Surg. (2018) 70:23-31. doi: 10.1007/s13304-018-0519-3

17. Zhou YY, Du XJ, Zhang CH, Aparicio T, Zaanan A, Afchain P, et al. Comparison of three lymph node staging schemes for predicting the outcome in patients with small bowel adenocarcinoma: a population-based cohort and international multicentre cohort study. EBioMedicine. (2019) 41:27685. doi: 10.1016/j.ebiom.2019.02.043

18. Guo QH, Zhu J, Wu XH, Wen H, Xia L, Ju X, et al. Validation of the prognostic value of various lymph node staging systems for cervical squamous cell carcinoma following radical surgery: a single-center analysis of 3,732 patients. Ann Transl Med. (2020) 8:485. doi: 10.21037/atm.2020.03.27

19. Cronin KA, Ries LA, Edwards BK. The Surveillance, Epidemiology, and End Results (SEER) program of the National Cancer Institute. Cancer. (2014) 120(Suppl. 23):3755-7. doi: 10.1002/cncr.29049

20. Wright JD, Lewin SN, Barrena Medel NI, Sun X, Burke WM, Deutsch I, et al. Endometrial cancer in the oldest old: tumor characteristics, patterns of care, and outcome. Gynecol Oncol. (2011) 122:69-74. doi: 10.1016/j.ygyno.2011.02.040

21. Patrizia DM, Jessica O, Giorgia M, Emanuela R, Davide F, Riccardo $\mathrm{V}$, et al. Surgical treatment of elderly patients with endometrial cancer ( $\geq 65$ years). J Geriatr Oncol. (2013) 4:368-73. doi: 10.1016/j.jgo.2013. 07.001

22. Wang J, Hassett JM, Dayton MT, Kulaylat MN. The prognostic superiority of log odds of positive lymph nodes in stage III colon cancer. J Gastrointest Surg. (2008) 12:1790-6. doi: 10.1007/s11605-008-0651-3

23. Bi G, Lu T, Yao G, Bian Y, Zhao M, Huang Y, et al. The prognostic value of lymph node ratio in patients with N2 stage lung squamous cell carcinoma: a nomogram and heat map approach. Cancer Manag Res. (2019) 11:942737. doi: 10.2147/CMAR.S216532
24. Yang M, Zhang H, Ma Z, Gong L, Chen C, Ren P, et al. Log odds of positive lymph nodes is a novel prognostic indicator for advanced ESCC after surgical resection. J Thorac Dis. (2017) 9:1182-9. doi: 10.21037/jtd.2017.03.187

25. Zhao C, Luo Q, Xia X, He F, Peng F, Yu X, et al. Risk score to predict mortality in continuous ambulatory peritoneal dialysis patients. Eur J Clin Invest. (2014) 44:1095-103. doi: 10.1111/eci.12344

26. Harrell FE Jr, Lee KL, Mark DB. Multivariable prognostic models: issues in developing models, evaluating assumptions and adequacy, and measuring and reducing errors. Stat Med. (1996) 15:361-87. doi: $\quad 10.1002 /($ SICI)1097-0258(19960229)15:4 < 361::AID-SIM168 $>3.0 . \mathrm{CO} ; 2-4$

27. Alba AC, Agoritsas T, Walsh M, Hanna S, Iorio A, Devereaux PJ, et al. Discrimination and calibration of clinical prediction models: users' guides to the medical literature. JAMA. (2017) 318:1377-84. doi: 10.1001/jama.2017.12126

28. SGO Clinical Practice Endometrial Cancer Working Group, Burke WM, Orr J, Leitao M, Salom E, Gehrig P, et al. Endometrial cancer: a review and current management strategies: part I. Gynecol Oncol. (2014) 134:38592. doi: 10.1016/j.ygyno.2014.05.018

29. Guo JB, Qian HL, Ma F, Zhang Y, Cui X, Duan H. The characteristics of isolated para-aortic lymph node metastases in endometrial cancer and their prognostic significance. Ther Adv Med Oncol. (2020) 12:18. doi: $10.1177 / 1758835920933036$

30. Pölcher M, Rottmann M, Brugger S, Mahner S, Dannecker C, Kiechle M, et al. Lymph node dissection in endometrial cancer and clinical outcome: a population-based study in 5546 patients. Gynecol Oncol. (2019) 154:6571. doi: 10.1016/j.ygyno.2019.04.002

31. Fleming ND, Frumovitz M, Schmeler KM, dos Reis R, Munsell MF, Eifel PJ, et al. Significance of lymph node ratio in defining risk category in node-positive early stage cervical cancer. Gynecol Oncol. (2015) 136:4853. doi: 10.1016/j.ygyno.2014.11.010

32. Ataseven B, Grimm C, Harter P, Prader S, Traut A, Heitz F, et al. Prognostic value of lymph node ratio in patients with advanced epithelial ovarian cancer. Gynecol Oncol. (2014) 135:435-40. doi: 10.1016/j.ygyno.2014.10.003

33. Bao X, Chen F, Qiu Y, Shi B, Lin LS, He BC. Log odds of positive lymph nodes is not superior to the number of positive lymph nodes in predicting overall survival in patients with oral squamous cell carcinomas. J Oral Maxillofac Surg. (2020) 78:305-12. doi: 10.1016/j.joms.2019.09.026

34. Agalar C, Aysal A, Unek T, Egeli T, Ozbilgin M, Akturk N, et al. The role of $\log$ odds of positive lymph nodes in predicting the survival after resection for ampullary adenocarcinoma. Pathol Oncol Res. (2020) 26:46773. doi: 10.1007/s12253-019-00584-6

Conflict of Interest: The authors declare that the research was conducted in the absence of any commercial or financial relationships that could be construed as a potential conflict of interest.

Copyright (c) 2021 Yang, Huang, Wang, Lai and Wu. This is an open-access article distributed under the terms of the Creative Commons Attribution License (CC BY). The use, distribution or reproduction in other forums is permitted, provided the original author(s) and the copyright owner(s) are credited and that the original publication in this journal is cited, in accordance with accepted academic practice. No use, distribution or reproduction is permitted which does not comply with these terms. 